\title{
EDITORIAL
}

\section{Process Chemistry in API Development}

\begin{abstract}
The timely supply of high-quality active pharmaceutical ingredients (APIs) depends on efficient process chemistry run under Good Manufacturing Practice (cGMP) guidelines. The enterprise requires the harmonization of technical, logistic, economic, and regulatory factors to result in a market viable production. This special of CHIMIA focuses on the technical and chemical aspects of API production known as Process Chemistry. Eighteen submissions outline a broad spectrum of topics in Process Chemistry: 1) company strategies in planning operations; 2) API target and chemical route evaluation; 3) chemical solutions to specific API targets; 4) technological methods for improving synthetic efficiency; 5) analytical methods for assessing safety in chemical operations; 6) educational opportunities at the academic/industry interface. Few of these topics arise in the normal course of chemical studies at university, but all of them become frontline concerns to the chemist entering the job market. It is our hope to highlight Process Chemistry and its challenges here in a way that brings home the excitement of this important pursuit.
\end{abstract}

Keywords: API production · Kilogram scale $\cdot$ Process chemistry

Drug discovery driven by the pharmaceutical industry be it chemical entities or biologics has been all the rage for the past few decades. Established companies and start-ups financed by Venture capital have searched intensively with the hope of finding that miracle cure for cancer, AIDS, diabetes or other major health problem. Ultimately, from a high-throughput library screen, the extract of a marine or terrestrial organism, or a directed medicinal chemistry structure-activity study a drug candidate emerges, the active pharmaceutical ingredient (API). This issue of CHIMIA covers mainly Process Chemistry revolving around small-molecule APIs, compounds with a molecular weight on the order of $500 \mathrm{AMU}$, embodying some stereochemical complexity. Assuming a dose of only $10 \mathrm{mg}$ per day would imply that for chronic treatment of 1 million patients the yearly amount of API needed is around four tons. When one considers the scale of such production, the importance of process chemistry is striking, more so if a synthetic procedure runs over multiple chemical conversions.

Synthetic procedures from medicinal chemistry studies are rarely suited for such commercial scale production. Even less well suited for production are the methods used to produce chemical libraries. Indeed, until the 'block buster' candidate has been identified, efficient chemical synthesis is at best a secondary concern. The reasoning is clear: the medicinal chemist needs access to a multitude of drug candidates for primary selection. Targeting a specific compound needs then a different approach. In addition as a new chemical entity (NCE) product the price of the API at the beginning of the drug development process plays a relatively small role. Therefore, the defining chemical question is "whether the API can be produced" at all, considerations of what that it will cost lag far behind. From a technical perspective one could say that detailed chemistry only gets attention if the supply line fails.

For a drug in pipeline the process needs to be 'workable' and reliable but not optimal. Of course, regulatory issues come into play too, wherein a high value is placed on reproducibility and readily documentable procedures. Factors of (supply) safety, purity and moderate robustness dominate the decision making for such chemical processes. Focusing on these factors means that one can often proceed to late clinical phases with a 'workable' or 'stable' procedure even if it is nowhere near the 'best possible'. Costs of the API are normally at most a few percent of the daily treatment costs and are hardly considered relevant at this point in the development.

Such 'workable' processes are often material inefficient, but once validated are hard to justify changing. If one focuses just on the cost of patent API chemical production one sees that it is relatively expensive compared to chemical commodities that need to be competitive. API 'workable' processes are relatively high input and low output (material and conversion ) with input/output-factors of 20 to even 100 per step performed; this assessment does not even consider, for example, the water consumption for cooling or extraction processes. It is obvious that good process solutions could lower costs by substantial factors. Smart companies therefore involve intelligent process chemistry from the beginning, sometimes even working on better and shorter alternative routes during the first phases of clinical development.

The cost of the process finally takes on new meaning when patents run out and generic production is contemplated. At this point, the typical per kilo market value for a given API substance drops substantially. This economic factor creates a need for more efficient chemistry, a good example of how the market promotes 'greenness' in chemical production.

New technologies also play an important role in streamlining processes. In the last couple of decades a number of new technological methods have been developed to help the process development chemist. Differential Scanning Calorimetry (DSC), PAT, parallel synthesis, accurate reaction calorimetry, in situ spectral observation via ReactlR, advanced crystallization techniques, microreactor technology, analytical liquid chromatographic methods (HPLC, LC-MS, Chiral LC), among others have become 'tools of the trade'. 
Statistics show an average of only $18 \%$ survival of drug candidates from clinical phase 1 to registration, therefore the pursuit of a full chemical process optimization may be wasted. This risk has to be accepted. It is eased by the fact that alternative processes in many cases deliver even research quantities faster and at much lower prices than the original process. So the venture into early optimization often pays off. Clearly, when market issues favor optimizing time to launch over good process chemistry then the short-term gain from rapid deployment will be favored over finely tuned process optimization. As API costs become more significant and margins get tighter then better and faster ways to optimal chemical processes become of paramount importance.

These economic or business chemistry issues are mostly alien to the academic chemist, especially those who do not consult for industries dealing with such large-scale production issues. It was that point which motivated us to co-edit this special issue of CHIMIA. Pleasantly, the general awareness of Process Chemistry is increasing and it is noteworthy that as we go to press with this issue, the American Chemical Society journal Chemical Reviews has just released its own issues dedicated to Process Chemistry. Therein Mark Lipton of Schering-Plough and Tony Barrett of Imperial College have assembled some 15 reviews in the area. Combined with their collection the present CHIMIA issue of 18 articles has even greater impact. An important point here is that although the area of Process Chemistry has a strong impact on the economic and technical nature of chemistry, the teaching of these economic and technical aspects in any formal way is extremely rare. Several excellent books on process chemistry exist, and the ACS journal Organic Chemistry Process and Development is dedicated to the field, still the academic/industry interface is not well integrated.

The University of Zurich and Siegfried have worked together to found a biannual Award in Process Chemistry: The Siegfried Medal and Symposium. The second of these will take place September 21st, 2006, contemporary with the release of this issue. Awardees come from industry and academics. The first award in 2004 was given to Pfizer's Joel Hawkins and the 2006 award goes to MIT's Stephen J. Buchwald.

In addition to these ceremonial activities, the University of Zurich offers a program in Business Chemistry as part of its normal curriculum and operates an industry/academic joint venture in the Laboratory for Process Research (know as LPF from its German name Laboratorium für Prozessforschung). Such formal teaching activities dramatically help bridge the academic/industry gap and the article by LPF's lead chemist Thomas Bader details the history of LPF, its unique scholar/professional mission, and some technical aspects of the LPF operation.

The general structure of the issue follows the Process Chemistry aspects of strategies, processes and technologies. Obviously any given article can present more than one of these, but normally with a focus on one. The first three articles come from Wyeth, Siegfried, and Lonza and have a significant strategic component. Bader's article on LPF follows with the academic/industry perspective. Then come eight solid examples of current Process Chemistry solutions to APIs of great or potential commercial interest. The colleagues from Roche, Pfizer, Merck, Givaudan, Boehringer-Ingelheim, Lilly, Chiron, and Dr Reddys present us with a spectrum of synthetic methods ranging from stereoselective catalytic methods to detailed reaction parameter optimization. The next five articles elaborate some of the technologies available in modern process chemistry. Aspects of finishing technologies like controlled crystallization from Novartis, calorimetry from the ETH-Zurich, microreactors from IMM-Mainz and SIAL, and simulated moving bed chromatography from SIAL all give an impression of the new technological directions for Process Chemistry. A final article from Nathaniel Finney at the Organic Chemistry Institute in Zurich closes our issue with a description of how the academic institution provides a special way to support industry development through academic freedom and innovation.

Overall, we hope that this issue is informative and entertaining in the sense that it creates an appreciation and further curiosity in the field of process chemistry. It has been our pleasure to put this together and we would like to thank again all of the contributors as well as the staff of CHIMIA for making this possible.

Hans-Ruedi Marti

Siegfried Ltd

Untere Brühlstrasse 4

$\mathrm{CH}-4800$ Zofingen

E-Mail: hansrudolf.marti@siegfried.ch
Jay S. Siegel

Organic Chemistry Institute, University of Zurich, Winterthurerstrasse 190

$\mathrm{CH}-8057$ Zurich

E-Mail: jss@oci.unizh.ch

\section{Suggested additional reading:}

Special Issue on 'Process Chemistry', Eds. M.F. Lipton, A.G.M. Barrett, and J. Michl, Chemical Reviews 2006, 109, $2581-3027$.

Neal G. Anderson, 'Practical Process Research \& Development' Academic Press, San Diego, 2000, pp 354.

'Process Chemistry in the Pharmaceutical Industry', Ed. K. Gadamasetti, Marcel Dekker, NY, 1999, pp 474.

Organic Process Research and Development (ACS Journal), http://pubs.acs.org/journals/oprdfk/index.html 